Friday 8 September 2017 


\title{
The Magnetoionic Universe: Timing, Bursts, and SETI
}

\author{
James M. Cordes \\ Astronomy Department and Center for Astrophysics and Planetary Science \\ Cornell University, Ithaca, NY U.S.A. 14853
}

\begin{abstract}
Propagation effects have been central to pulsar research and indeed were an integral part of the pulsar discovery and its prologue. I will summarize the early deduction process for establishing pulsar distances and refinements to the distance scale and modeling of the Galaxy in electron density and magnetic field. This will lead to the analogous current situation of understanding distances and media for extragalactic radio bursts. The role of magnetoionic media in precision pulsar timing and surveys for transients will be summarized. Finally, going full circle, searches for extraterrestrial intelligence (LGMs) also require attention to propagation effects.
\end{abstract}

Keywords. Magnetic fields, pulsars: general, interplanetary medium, ISM:magnetic fields, ISM: structure, intergalactic medium

\section{Introduction}

The discovery of pulsars in 1967 was both a consequence and a tremendous stimulus of studies of wave propagation through magnetic ionized gas. Intensity scintillations were identified in the early 1950s along with the establishment of interplanetary scintillations (IPS) in the middle of the decade. This led to the construction of the Cambridge IPS telescope to find compact sources $(\lesssim 1$ arcsec) that would be good candidate quasistellar objects (quasars) and accompanied lunar occultations as a means for finding and localizing compact sources. As a parallel effort in this quest for angular resolution, very long baseline interferometry was also developed in the mid-1960s. The discovery of pulsars opened the door to unique studies of the interstellar medium that continues to the present time. These include development of the pulsar distance scale along with a Galactic model for the free electron density, establishment of turbulence (or processes that mimic it) as the underlying cause of small-scale $\left(\lesssim 10^{4} \mathrm{~km}\right.$ density variations that underly interstellar scintillation (ISS), and detailed mapping of the Galaxy's magnetic field strength. These developments are portrayed in the time line shown in Figure 1. The figure identifies some of the names associated with the developments of the 1950s and 1960s but is by no means complete.

The figure also advances to the present time when fast radio bursts (FRBs) have garnered our attention. These are radio flashes of $\sim 0.1$ to $10 \mathrm{~ms}$ durations that evidently originate in other galaxies. The establishment of the FRB distance scale is still incomplete but the redshift $(z=0.19)$ of the repeating FRB 121102 demonstrates the extragalactic location of this source. For all of the other FRBs (to date), their extragalactic distances rely on the removal of the foreground electron column density estimated from the pulsar based models. 


\section{Scintillations and Sources}

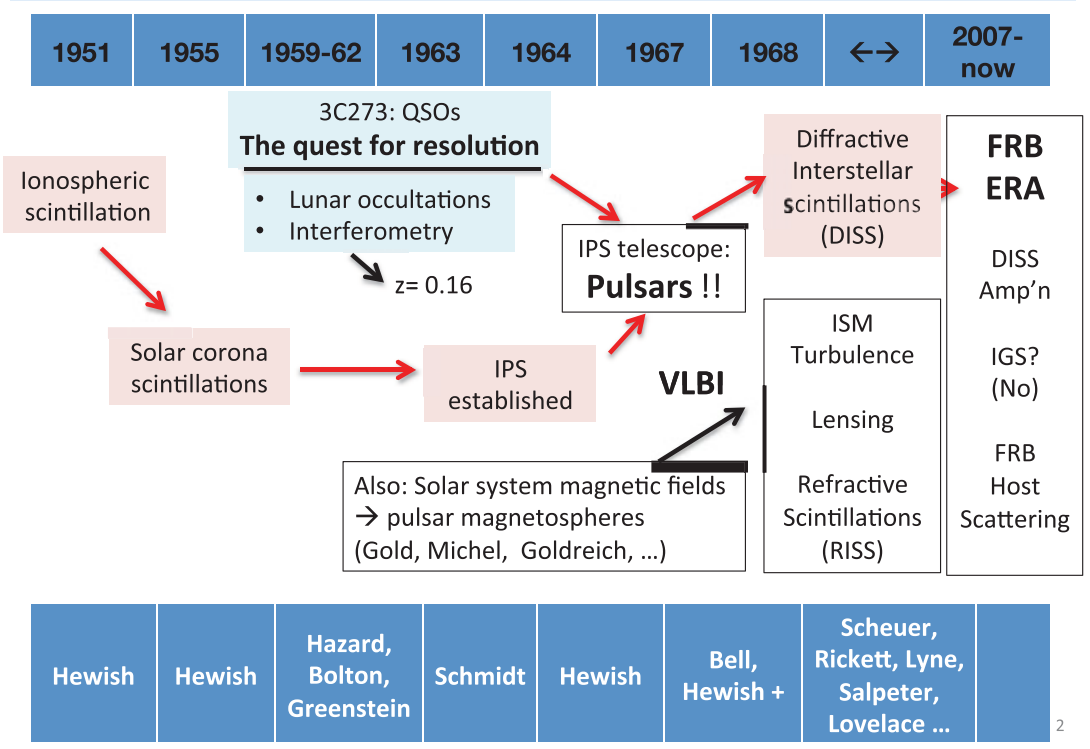

Figure 1. A topical history of developments leading to the discovery of pulsars and detailed studies of the interstellar medium. Extragalactic bursts have captured the attention of astronomers in recent years and have raised many of the same questions that were raised in the immediate post-discovery years of pulsars.

\section{Dispersion Laws and Distances}

The pulsar discovery paper Hewish et al. (1968) estimated distances of the 'pulsating sources' by interpreting the measured frequency sweep rate in terms of that for a tenuous, unmagnetized plasma ( $c=$ speed of light, $\nu=$ frequency, $d=$ distance, and $n_{\mathrm{e}}=$ electron density),

$$
\frac{d \nu}{d t}=-\frac{c \nu^{3}}{d \nu_{p}^{2}} \propto \frac{\nu^{3}}{d n_{\mathrm{e}}} .
$$

Electron densities were poorly known at the time and the adopted value of $0.1 \mathrm{~cm}^{-3}$ yielded distances of tens of parsecs, substantially smaller than current parallax distances. It was rapidly realized that pulse arrival times (TOAs) could further constrain the physics of pulsars and their sight lines. Extending the analysis of Tanenbaum et al. (1968), the dispersion, emission, and Faraday rotation measures (with the parallel magnetic field $B_{\|}$ in $\mu G)$,

$$
\mathrm{DM}=\int_{0}^{\mathrm{d}} \mathrm{dsn}_{\mathrm{e}} \quad \mathrm{EM}=\int_{0}^{\mathrm{d}} \mathrm{dsn}_{\mathrm{e}}^{2} \quad \mathrm{RM}=0.81 \int_{0}^{\mathrm{d}} \mathrm{ds} \mathrm{n}_{\mathrm{e}} \mathrm{B}_{\|},
$$

yield a compact expression for the frequency dependence of the arrival time of the two hands of circular polarization,

$$
t(\nu)=k_{1} \frac{\mathrm{DM}}{\nu^{2}} \pm k_{2} \frac{\mathrm{RM}}{\nu^{3}}+k_{3} \frac{\mathrm{EM}}{\nu^{4}},
$$

where $k_{1}=4.15 \mathrm{~ms}, k_{2}=0.18 \mathrm{~ns}$, and $k_{3}=0.25 \mathrm{ps}$ for DM in pc cm ${ }^{-3}, \mathrm{RM}$ in $\mathrm{rad} \mathrm{m}^{-2}$, $\mathrm{EM}$ in $\mathrm{pc} \mathrm{cm}^{-6}$, and $\nu$ in GHz. No departures from the $\nu^{-2}$ term were seen, indicating that stellar coronae or highly magnetized thermal (hydrogen) plasmas were not involved. 
The DMs of FRBs are larger by significant factors than the Galactic contributions estimated from density models [Cordes \& Lazio (2002), Yao et al. (2017)], indicating that they are extragalactic. Recent work on FRBs has also tested for departures from the simplest dispersion law, without success, again implying that nowhere along FRB lines of sight is there a high electron density. DMs of FRBs therefore receive contributions from the Galaxy, the intergalactic medium (IGM), and the host galaxies. In the case of FRB121102, these contributions are all roughly equal [Tendulkar et al. (2017)].

\section{Magnetic Fields}

Early work by Smith (1968) and Manchester (1972) showed that linearly polarized pulsar radiation could be used to determine RM and the average line of sight magnetic field $B_{\|}=\mathrm{RM} / 0.81 \mathrm{DM}$. It was also established that there was no contribution to RM from the pulsar itself. Today this is understood to be a consequence of the normal modes being linear inside the pulsar magnetosphere combined with the high relativistic particle energies and the plasma likely comprising $e^{ \pm}$pairs rather than a hydrogen plasma.

In recent years, pulsar RMs have been combined with a much larger number of extragalactic RMs to infer the structure of the Milky Way's magnetic field. There is at least one major reversal of the magnetic field in the inner Galaxy and the field generally seems to follow spiral arms [Han (2013)]. Future surveys with the Jansky Very Large Array, MeerKAT, and the Square Kilometer Array will allow even greater resolution of Galactic features and perhaps a more definitive analysis of the spiral arm structure than exists today.

\section{Galactic Electron Density Models and Spiral Structure}

The large number of current pulsar DMs ( 2600) along with distance constraints from HI absoprtion; associations of pulsars with globular clusters, supernovae, and companion white dwarfs; and direct parallax measurements from timing or interferometry provide the input needed for fairly detailed models of the Galactic electron density. However, only about $5 \%$ of pulsars have useful distance constraints and relatively small scale structures in the Galaxy, especially HII regions, require a much larger number of pulsars to sample them. Consequently, mathematical models for spiral arms and disk components have been the basis for a parametric approach. At present even the number of spiral arms in the Milky Way and their locations and extents are still under debate [Churchwell et al. (2009), Reid et al. (2009)]. As the number of pulsars lines of sight grows and as distances to HII regions are better measured, it may be possible to take a different approach to spiral arm components in electron density models: use the HII regions directly to model the electron density. This approach could replace the current one of using HII regions to define (some) properties of smooth spiral arm models. Instead, the HII regions can 'speak for themselves.' A new model that will soon replace the NE2001 model [Cordes \& Lazio (2002)] is being developed using the most up to date parallaxes and using alternative methods for defining spiral arms.

\section{Pulse and Amplitude Fluctuations: then and now}

Early work on pulsars contrasted the stability of pulse periods against the volatility of pulsar amplitudes on a variety of time scales. The discovery paper [Hewish et al. (1968)] hypothesized that short term variations (seconds) might be due to IPS and the longer ones from variations in the ionospheric RM. However, it was rapidly sorted out that the 


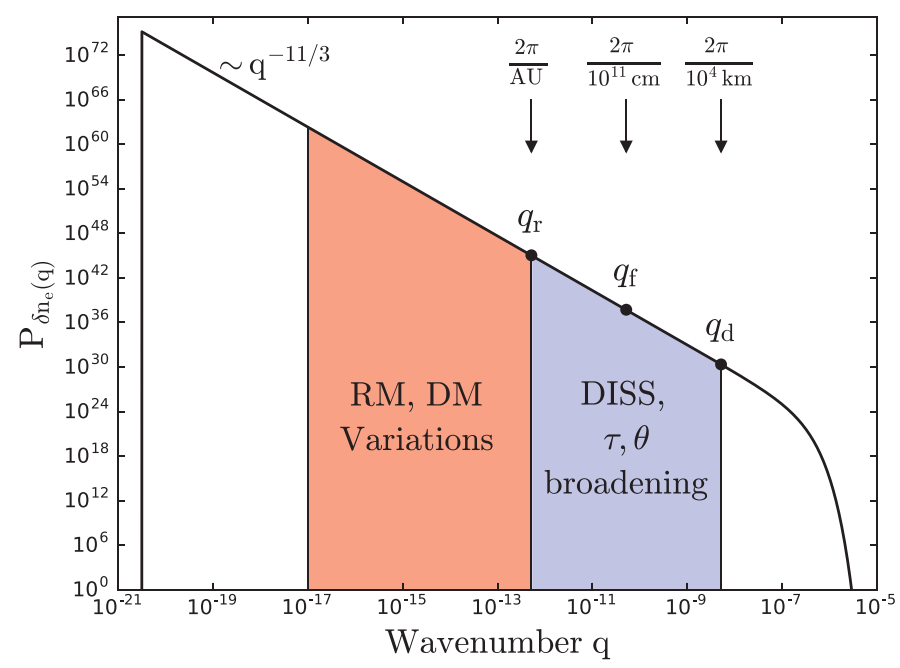

Figure 2. A cartoon spectrum of electron density variations in the Galaxy derived from diffractive scintillation and pulse broadening, DM variations in time, and RM variations. Labeled wavenumbers are $q_{\mathrm{d}}=$ wavenumber corresponding to diffractive scintillations, $q_{\mathrm{f}}=$ Fresnel wavenumber, and $q_{\mathrm{r}}=$ refractive scintillation wavenumber. These correspond to length scales of $1 \mathrm{AU}, 10^{11} \mathrm{~cm}$, and $10^{4} \mathrm{~km}$, respectively. For a Kolmogorov spectrum with wavenumber index of $11 / 3$, we have $q_{\mathrm{d}} q_{\mathrm{r}}=q_{\mathrm{f}}^{2}$. Refractive scintillations are associated with wavenumbers $q \lesssim q_{\mathrm{r}}$.

rapid variations were intrinsic to pulsars while interstellar scintillations were responsible for longer variations on time scales of minutes to hours [Pilkington et al. (1968), Lyne \& Rickett (1968), Scheuer (1968), Salpeter (1969)]. Significantly later, in the 1980s [Rickett et al. (1984)], refractive scintillations were identified as the cause of much slower intensity variations than the faster diffractive scintillations first identified.

Since 1968, much effort on pulsar amplitude variations vs. time and frequency has identified that a very wide range of length scales is present in the electron density, ranging from a few hundred kilometers to hundreds of parsecs. In particular, it has been posed that there is a 'grand power law in the sky,' i.e. a power-law wavenumber spectrum for $n_{e}$ that spans many orders of magnitude and has a slope in wavenumber space similar to that of the Kolmogorov spectrum, wavenumber ${ }^{-11 / 3}$.

Figure 2 shows a notional spectrum for $\delta n_{\mathrm{e}}$ that is of the form $P_{\delta n_{\mathrm{e}}}(q)=\mathrm{C}_{\mathrm{n}}^{2} \mathrm{q}^{-\beta}$ with $\beta \approx 11 / 3$ (the Kolmogorov index). A large body of measurements allows estimation of the spectral coefficient $\mathrm{C}_{\mathrm{n}}^{2}$ and $\beta$, with mixed outcomes. While there are departures from an $11 / 3$ spectrum, these may be due simply to deterministic features (sheets and filaments) that superpose with a genuine turbulence spectrum or to confinement of turbulent fluctuations in 'containers' whose size affects the scaling laws expected from indefinitely distributed turbulence. Corroborating evidence for the existence of a broad power-law density spectrum is that cosmic propagation in the Galaxy requires magnetic fluctuations $\delta B$ on a similar range of length scales and that $\delta n_{\mathrm{e}} / n_{\mathrm{e}} \sim \delta B / B$.

A worthy quote from Scheuer's 1968 paper has current relevance:

The rapid ... fluctuations appear to be well correlated on different frequencies and ... are probably an intrinsic property of the source. The long term variations show little, if any, correlation between different frequencies ... Such complex frequency structure suggests that the emission process is modulated by interference phenomena, either between a number of coherent pulse sources on the surface of a star, or else between 


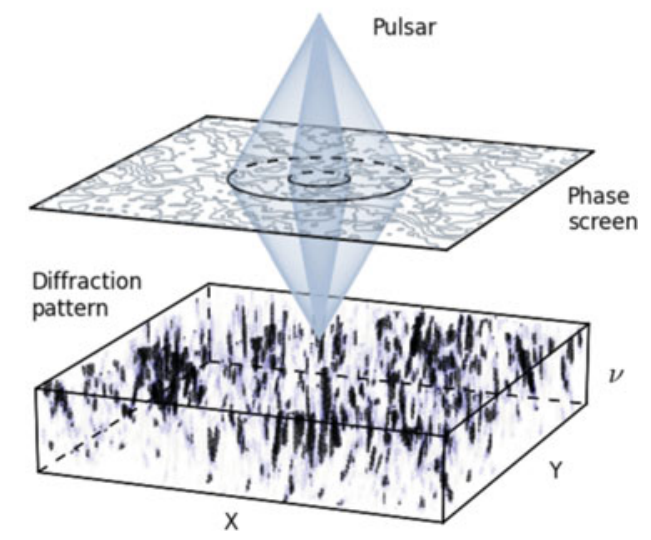

Figure 3. Multipath scattering from a thin phase screen. The cones indicate scattering at lower (wide cone) and higher frequencies. The dark elongated features in the rectangular slab indicate intensity maxima in the diffraction pattern produced by the screen. These 'scintles' have characteristic spatial and frequency scales. The line of sight cuts through this pattern by virtue of the motions of the pulsar, screen and observer. For high field pulsars $\left(\sim 10^{12} \mathrm{G}\right)$ the motion is dominated by the pulsar while for millisecond pulsars the Earth's orbital motion also is significant.

a number of images of the source caused by irregular refraction in an interstellar medium.

FRBs indeed show complex frequency structure that is not yet understood and the debate continues between intrinsic vs. extrinsic causes much like that of 50 years ago!

\section{Timing}

Pulsar timing has developed into a hard science that links neutron star physics, interstellar propagation, and solar system ephemerides via their contributions to the error budget of timing precision on any pulsar. Interstellar propagation of course enters front and center into timing precision. Figure 3 shows diffractive intensity structure vs. $X-Y$ spatial coordinates and frequency $\nu$.

Multipath propagation from the pulsar to us causes several effects: (1) temporal variations in dispersion measure, $\mathrm{DM}(\mathrm{t}) ;(2)$ diffractive intensity scintillations vs. time and frequency; and (3) frequency dependence of DM. Analysis of $\mathrm{DM}(\mathrm{t})$ has been used to constrain the wavenumber spectrum. Diffractive scintillations cause $100 \%$ excursions in intensity that can strongly affect timing precision, which depends on the signal-to-noise ratio of pulse measurements. Additionally, diffractive scintillations across a receiver band couple with the frequency dependence of pulsar pulse profiles to produce a stochastic variation in TOA. These can be mitigated by appropriate weighting of the measurements vs. frequency. The third effect, frequency dependence of DM may pose a limitation in timing precision at the tens of ns level that may be important in the usage of millisecond pulsars to detect gravitational waves [Cordes et al. (2016)].

\section{FRBs, Scintillations, and Lensing}

FRBs are short enough that diffractive interstellar scintillations (DISS) from the Milky Way are expected unless extragalactic scattering, which is identified as asymmetric 
temporal broadening, causes the source to appear too large to the turbulence in the Galaxy. In fact, DISS has been explicitly identified in two FRB sources, so it is reasonable to expect that it also occurs in most FRBs. In that case, the exponential distribution of DISS modulations is highly significant because if there are many FRB sources producing many bursts, it is possible that the only detectable bursts are those that have been scintillated into a very high intensity state. With sufficient statistical trials, it is reasonable to expect at least one order of magnitude of modulation. This has two implications: FRB detections are only the tail of the distribution of events and because such large DISS excusions are rare, any given FRB may repeat only rarely. The repeating FRB 121101 is a clear counter example, but its low Galactic latitude in the anticenter direction implies that DISS could not have played a role in its initial detection and therefore also not in its repetitions.

Lensing from discrete plasma structures produces even greater intermittency than DISS. Very large gains up to 100 can be expected along with multiple images that may produce multiple bursts from a single event and may introduce interference from multiple bursts that overlap in arrival time.

\section{The Search for Extraterrestrial Intelligence (SETI)}

SETI often targets narrowband transmissions from ETs (LGMs). Because transmitters are small, DISS of SETI signals should occur and it will produce the same intermittency seen from pulsars and expected from FRBs. If SETI is ever successful, its methodology will likely have been highly informed by what we have learned from pulsars, the original 'LGM' sources.

\section{Acknowledgement}

I thank many colleagues for inspiration, collaboration, and friendship over the years since I got into the pulsar business as a latecomer to the party in 1971: T. Hankins, B. Rickett, J. Weisberg, J. Rankin, J. Taylor, I. Wasserman, M. McLaughlin, S. Chatterjee, and R. Shannon. This work was partially supported by the National Science Foundation (NSF) Physics Frontier Center award number 1430284.

\section{References}

Cordes, J. M. \& Lazio, T. J. W. 2002, arXiv:astro-ph/0207156

Cordes, J. M., Shannon, R. M., \& Stinebring, D. R. 2016, ApJ, 817, 16

Churchwell, E., Babler, B. L., Meade, M. R., et al. 2009, PASP, 121, 213

Han, J. 2013, Solar and Astrophysical Dynamos and Magnetic Activity, 294, 213

Hewish, A., Bell, S. J., Pilkington, J. D. H., Scott, P. F., \& Collins, R. A. 1968, Nature, 217, 709

Lyne, A. G. \& Rickett, B. J. 1968, Nature, 218, 326

Manchester, R. N. 1972, (ApJ), 172, 43

Pilkington, J. D. H., Hewish, A., Bell, S. J., \& Cole, T. W. 1968, Nature, 218, 126

Reid, M. J., Menten, K. M., Zheng, X. W., et al. 2009, ApJ, 700, 137-148

Rickett, B. J., Coles, W. A., \& Bourgois, G. 1984, A\&SA, 134, 390

Salpeter, E. E. 1969, Nature, 221, 31

Scheuer, P. A. G. 1968, Nature 218, 920

Smith, F. G. 1968, Nature, 218, 325

Tanenbaum, B. S., Zeissig, G. A., \& Drake, F. D. 1968, Science, 160, 760

Tendulkar, S. P., Bassa, C. G., Cordes, J. M., et al. 2017, ApJL, 834, L7

Yao, J. M., Manchester, R. N., \& Wang, N. 2017, ApJ, 835, 29 